\title{
Lymphadenopathy with Polyclonal Hypergammaglobulinemia
}

National Cancer Institute

\section{Source}

National Cancer Institute. Lymphadenopathy with Polyclonal Hypergammaglobulinemia.

NCI Thesaurus. Code C9307.

A finding indicating the presence of enlarged lymph nodes and associated polyclonal

hypergammaglobulinemia. 